Revisão

Review

\title{
Exercício físico e CoViD-19: aspectos de saúde, prevenção e recuperação: uma breve revisão narrativa
}

\section{Exercise and CoViD-19: Health, Prevention and Recovery Aspects: A Brief Narrative Review}

\author{
Lilian Martins ${ }^{1,2} \mathrm{PhD}$; Renato Soeiro ${ }^{1} \mathrm{MSC}$
}

Recebido em: 08 de desembro de 2020. Aceito em: 10 de dezembro de 2020.

Publicado online em: 29 de dezembro de 2020.

DOI: $10.37310 /$ ref.v89i4.2724

\begin{abstract}
Resumo
Introdução: 0 novo coronavírus, agente etiológico da CoViD-19, causa danos a diversos sistemas do corpo humano, particularmente, aos pulmões, coração, cérebro, rins e sistema vascular. Tendo sido espalhada para o mundo no início de 2020, pouco se sabia sobre a doença. Mais recentemente, começaram a surgir evidências de que o exercício físico pode ajudar na sua prevenção.

Objetivo: Apresentar uma breve revisão da literatura acerca da relação de exercício físico com promoção da saúde do sistema imunológica e sua relação com a CoViD-19.

Métodos: Este estudo foi do tipo revisão narrativa. A busca foi realizada na base da dados do PubMed e o Google Scholar. A linguagem utilizada foi o inglês e foram utilizados os seguintes termos: "exercise and CoViD-19".

Resultados e Discussão: O exercício físico melhora a função endotelial, aprimorando o sistema imunológico, contribui para reduzir a obesidade, bem como contribui para melhorar a saúde mental, proporcionando benefícios adicionais, pois, tanto estados afetivos negativos quanto obesidade aumentam a inflamação e diminuem a função imunológica. Além disso, a literatura exibe dados que mostraram que maiores níveis de atividade física se

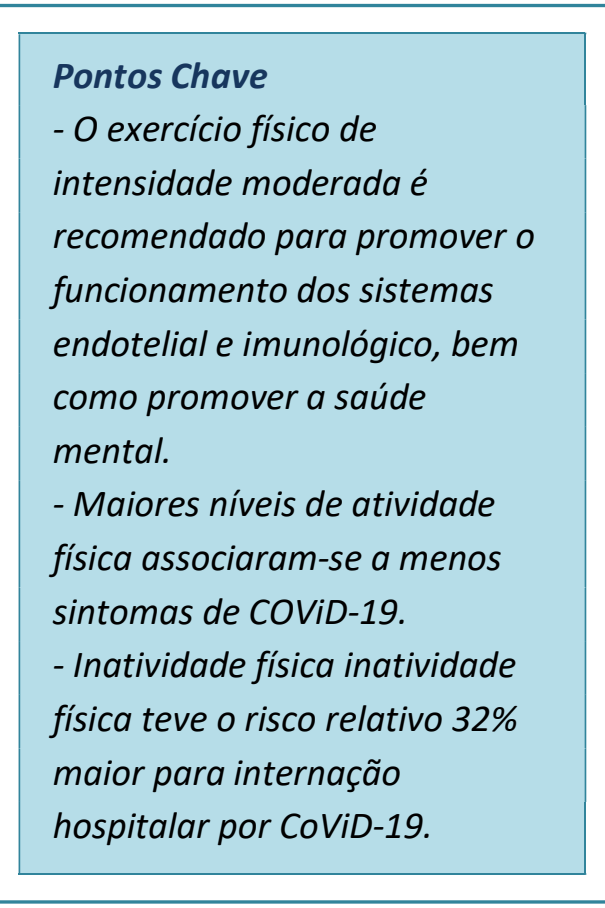
associaram a menor prevalência de sintomas de CoViD-19 e inatividade física teve o risco relativo 32\% maior para internação hospitalar por CoViD-19.

Conclusão: 0 exercício promove a saúde mitocondrial, contribuindo para a manutenção do sistema imunológico. A intensidade do exercício físico é importante para melhorar a função endotelial, a imunidade e a saúde mental. Assim o exercício deve ser de intensidade moderada, além de ser mantida a regularidade da prática para que os benefícios orgânicos sejam alcançados.
\end{abstract}

Palavras-chave: atividade física, imunologia, coronavírus, pandemia, medicina preventiva.

\footnotetext{
$\S$ Autor correspondente: Lilian Martins - e-mail: lilitina@gmail.com Afiliações: ${ }^{1}$ Instituto de Pesquisa da Capacitação Física do Exército (IPCFEx), Rio de Janeiro-RJ, Brasil; ${ }^{2}$ Laboratório de Mapeamento Cerebral e Integração Sensório-Motora (LABISM), Escola de Educação Física e Desportos (EEFD) da Universidade Federal do Rio de Janeiro (UFRJ), Rio de Janeiro-RJ, Brasil.
} 


\begin{abstract}
Introduction: The new coronavirus, the etiological agent of CoViD-19, causes damage to several systems in the human body, particularly the lungs, heart, brain, kidneys and vascular system. Spread to the world in early 2020, little was known about the disease. More recently, evidence has begun to emerge that physical exercise can help prevent it.

Objective: To present a brief review of the literature about the relationship between exercise and health promotion of the immune system and its relationship with CoViD-19.

Methods: This study was a narrative review. The search was carried out using the PubMed and Google Scholar databases. The language used was English and the following terms were used: "exercise and CoViD-19".

Results and Discussion: Exercise improves endothelial function, improving the immune system, contributes to reducing obesity, as well as contributing to mental health, providing additional benefits, as both negative affective states and obesity increase inflammation and decrease function immunological. In addition, the literature shows that higher levels of physical activity were associated with lower prevalence of CoViD-19 symptoms and physical inactivity had a 32\% higher relative risk for hospitalization for CoViD-19.

Key Points

- Exercise at moderate intensity

is recommended to improving

endothelial and immune

systems functioning, as well as

to better mental health.

- Higher levels of physical

activity were associated with

lower prevalence of CoViD-19

symptoms.

- Physical inactivity Physical

inactivity had a $32 \%$ higher

relative risk for hospitalization

for CoViD-19.

Conclusion: Exercise promotes mitochondrial health, contributing to the maintenance of the immune system. The intensity of physical exercise is important to improve endothelial function, immunity and mental health and must be moderate, in addition to maintaining the regularity of the practice so the organic benefits can be achieved.
\end{abstract}

Keywords: physical activity, immunology, coronavirus, pandemic, preventive medicine.

\title{
Exercício físico e CoViD-19: aspectos de saúde, prevenção e recuperação: uma breve revisão narrativa
}

\section{Introdução}

A doença causada pelo novo coronavírus pode levar à síndrome respiratória aguda grave ((Severe Acute Respiratory Syndrome: SARS). Nos anos de 2002-2003 houve uma pandemia de SARS causada, também, por um coronavírus, originário da China e o vírus foi denominado SARSCoV. Assim, na pandemia de 2020, causada pelo novo coronavírus que, também, causava SARS e tendo sua origem em 2019 na China foi denominado SARS-CoV2. A doença apresentou diversos sintomas que poderiam se agravar muito levando $o$ paciente à hospitalização e foi denominada CoViD-19 (do inglês: coronavirus disease in 2019). Provoca efeitos deletérios inflamatórios $(\mathbf{1 , 2})$ e neurológicos(3-7) e, em sua fase severa, desencadeia uma tempestade de citocinas(8), um fenômeno flogístico $^{1}$ - caracterizado por quadro inflamatório e febril, que é retro-alimentado pela produção de citocinas processo que sobrecarrega os mecanismos de contrarregulação $(\mathbf{8 , 1 0})$.

O presente estudo teve por objetivo apresentar uma breve revisão da literatura acerca da relação de exercício físico com aspectos de saúde, prevenção e recuperação da CoViD-19.

\section{Métodos}

Este estudo foi do tipo revisão narrativa. A busca foi realizada na base da dados do

\footnotetext{
${ }^{1}$ Flogístico: (med) inflamatório(9).
} 
PubMed, que inclui MedLine e outras bases científicas - sendo principal fonte de consulta científica na área das ciências da saúde, bem como no Google Scholar. Utilizou-se a língua inglesa para rastrear as publicações, cujos termos foram "exercise and CoViD-19".

\section{Resultados e Discussão}

\section{CoViD-19 uma doença do sistema endotelial}

O SARS-CoV2, agente etiológico da CoViD-19, causa danos a diversos sistemas do corpo humano, particularmente, aos pulmões, coração, cérebro, rins e sistema vascular(10). Isto se explica porque os receptores do SARS-CoV2 é a proteína ACE2, que se apresenta predominantemente nos alvéolos pulmonares e nas células epiteliais respiratórias, estando presente, ainda, no miocárdio, ílio, esôfago e no neuroepitélio olfatório(11). Nunn et al(11) explicam que o risco aumentado para o agravamento da CoViD-19 em obesos se deve ao fato de que os receptores ACE2 são encontrados no tecido adiposo: tanto no tecido visceral quanto no tecido subcutâneo. Os autores apontam que é provável que outros agentes - CD26 ou CD147, possam estar atuando, também, como receptores do SARS-CoV2, que são alterados com a idade, bem como em comorbidades como obesidade e hipertensão. CD147 e ACE2 apresentam-se aumentados em caso de doença pulmonar, que ativa o sistema renina-angiotensina aldosterona (reninangiotensin aldosterone system: RAAS), acelerando os danos no organismo o que, segundo os autores, explica a tempestade de citocina. Por esse motivo, o grau de severidade da CoViD-19 relaciona-se com a existência prévia de outras doenças relacionadas à idade como diabetes, hipertensão e outras doenças cardiovasculares(12,13), que comprometem a função endotelial.

As complicações da doença são mais frequentes em idosos porque com o avançar da idade, há redução da função pulmonar, que causa prejuízo da integridade epitelial pulmonar, comprometendo muito a capacidade dos idosos de combater infecções respiratórias, resultando em maior susceptibilidade a doenças pulmonares(14). Esse declínio da função respiratória relacionado à idade independe do exercício físico(15).

Libby e Lüscher(10) investigaram os mecanismos da CoViD-19 e examinando os estágios de complicações posteriores, observaram que se trata de uma doença endotelial. Os autores explicam:

"O endotélio vascular fornece a interface crucial entre o compartimento do sangue $e$ os tecidos, exibindo uma série de propriedades notáveis que, normalmente, mantêm a homeostase. Essa paleta de funções rigidamente regulada inclui o controle da hemostasia, fibrinólise, vasomoção, inflamação, estresse oxidativo e estrutura da permeabilidade vascular.

Embora essas funções participem momento-a-momento da regulação da circulação e da coordenação de muitos mecanismos de defesa do organismo, também podem contribuir para a ocorrência de doenças, nesse caso, quando as funções homeostáticas tornam-se funções defensivas e atingindo limites, afetam o próprio organismo. (...)

Citocinas, mediadores de proteínas pró-inflamatórias, servem como sinais de perigo chave que mudam as funções endoteliais do modo homeostático para o defensivo, para ao final, provocar uma tempestade de citocinas que sobrecarregam os mecanismos de regulação endotelial.

O conceito de COVID-19 como uma doença endotelial fornece um quadro fisiopatológico unificador desta infecção violenta e, ainda, provê uma estrutura para o desenvolvimento de estratégia para o tratamento (...).

\section{Exercício físico e saúde}

O exercício físico promove a saúde de vários sistemas do organismo humano: cardio-vascular(16-18), endócrino(12,19), 
psicoló-gico(20,21),

imunológico(11,20,22), respira-tório(23).

Tais benefícios abrangentes podem explicar a associação de exercício físico com prevenção de diversos tipos de cânceres(24).

Os efeitos do exercício físico que atingem todos os tecidos do organismo humano são os que ocorrem no endotélio vascular - que é a interface crucial entre o compartimento do sangue e os tecidos(10). Libby e Lüscher(10) explicam que o endotélio é a única superfície em que, em condições fisiológicas, o sangue se apresenta sem coagular. A prática de exercício físico, com o aumento de consumo de oxigênio, faz com que o sangue circule com um aumento da força tangencial do sangue que flui na superfície endotelial do vaso sanguíneo tensão de cisalhamento, em inglês: "shear stress"(25-28). O shear stress que ocorre durante o exercício promove a adaptação vascular, havendo até mesmo um remodelamento dos vasos $(\mathbf{2 9}, \mathbf{3 0})$, devido a esses estímulos hemodinâmicos. Tais alterações benéficas induzem mudanças funcionais e estruturais na parede arterial por meio da transdução do sinal das células endoteliais(31). Maiorana et al.(25) explicam que o shear stress nas células endoteliais é um estímulo potente para a produção de óxido nítrico (Nitric Oxid: NO), sendo que o treinamento físico envolvendo sessões repetitivas de exercícios durante semanas ou meses regula de modo a aumentar a bioatividade do NO endotelial. Nas palavras de Green e Smith(32): "exercise is vascular medicine" [exercício é medicina vascular].

Mais recentemente, os cientistas descobriram que o sistema mitocondrial é um componente fundamental para um sistema imunológico efetivo(11,33). As mitocôndrias são organelas altamente móveis devido à fissão ${ }^{2}$, fusão, transporte e mitofagia - processos que em conjunto são chamados de dinâmica mitocondrial. Essa

\footnotetext{
${ }^{2}$ Fissão: processo celular de autorreplicação(34).
}

dinâmica desempenha um papel importante em produção de energia, divisão celular, diferenciação celular e morte celular(33). Xie et al. (33), em estudo recente de revisão sistemática, explicaram que, na última década, muitos estudos demonstraram a importância do metabolismo mitocondrial no sistema imune, sendo que a dinâmica mitocondrial tem papel essencial nas respostas imunológicas, as quais são mediadas por vários tipos de células, como células imunes $\mathrm{T}, \mathrm{B}$ e outras células envolvidas na resposta imune inata. $\mathrm{O}$ controle da inflamação depende do controle das espécies reativas de oxigênio (reactive oxigen species: ROS) na mitocôndria. A saúde do sistema mitocondrial depende da hormese $^{3}$ mantendo o metabolismo alerta(11). Os fatores que promovem a hormese mitocondrial são exercício físico (em doses subletais), com-ponentes vegetais na dieta e restrição calórica(11,36,37). O exercício físico promove o efeito de hormese, sobretudo o do tipo aeróbico, favorecendo a função mitocondrial, tanto em jovens quanto em idosos(11). Nunn et al. (11) explicam que, por um lado, o músculo ativo, geralmente, apresenta-se em estado inflamado, que por outro lado, promove compensações ao organismo, induzindo mecanismos antiinflamatórios e antioxidantes poderosos. Os autores(11) apontam que o exercício físico, desde que não seja feito de modo excessivo, sobretudo permitindo tempo suficiente para a recuperação, é altamente benéfico, pois, melhora a resposta anti-inflamatória e antioxidante. Além disso, cientistas têm demonstrado que o músculo possui importantes funções imunológicas, abrigando e fornecendo células-tronco antivirais, evitando a exaustão de células $\mathrm{T}$ e protegendo o potencial proliferativo durante a inflamação $(\mathbf{1 1 , 3 8 )}$.

A literatura aponta que exercícios de intensidade moderada estimulam a

\footnotetext{
${ }^{3}$ Hormese: Resposta adaptativa de células e organismos a um estresse moderado (geralmente intermitente)(35).
} 
imunidade celular, enquanto os de alta intensidade, sem tempo de recuperação adequado disparam uma diminuição dessa imunidade(39).

\section{Atividade física, saúde mental e inflamação}

De acordo com estudos epidemiológicos longitudinais, conduzidos amostras populacionais, pessoas com inflamação aumentada têm risco maior para desenvolver doenças cardiovasculares(40), diabetes tipo 2(41) além de desordens psiquiátricas(42). A literatura mostra que estresse mental crônico e humor - fatores psicossociais são reconhecidos como importantes preditores de longevidade e bem-estar(43). Estudos mostram que a depressão está independentemente associada a doenças cardiovasculares, mortalidade por todas as causas e como comorbidade a doenças crônicas, sendo que podem piorar seus prognósticos(43). Sintomas neuropsiquiátricos e doenças mentais estão comumente presentes em pacientes com doenças sistêmicas crônicas, sendo que ansiedade e depressão, estão presentes em até $50 \%$ desses pacientes, resultando em recuperação física prejudicada e em regime de tratamento mais complexo(44). Além disso, o estresse associado aos aspectos físicos e emocionais da doença sistêmica possuem efeitos prejudiciais que dão origem a transtornos mentais comórbidos. Duric et al. (44) explicam que o estresse psicológico decorrente de estados afetivos negativos aumentam a inflamação e diminuem a função imunológica. Relatórios clínicos indicam que a relação entre doenças sistêmicas e psiquiátricas é bidirecional(44), isto é, tanto as doenças crônicas afetam a saúde mental, quanto a saúde mental pode estar associada ao desenvolvimento de doenças crônicas.

A literatura mostra que o exercício físico regular associa-se à redução do estresse psicológico e ao melhor humor e, por conseguinte, pode mediar, em parte, as associações entre depressão, estresse e desfechos em saúde(43,45-47). Nesse contexto, o exercício físico tem um papel importante na redução da inflamação, entretanto, a intensidade deve ser observada com atenção. Paolucci et al.(48), em estudo experimental, longitudinal (seis semanas) mostraram que o exercício ótimo para a promoção da saúde mental com diminuição da inflamação foi o exercício do tipo contínuo e de intensidade moderada.

\section{A relação entre atividade física e CoViD- 19}

Por um lado, a ciência avança conhecendo melhor os mecanismos da doença e por outro, vão surgindo novas evidências de que a atividade física pode contribuir para a prevenção da CoViD-19.

Silveira et al.(39) discutiram em profundidade como o exercício pode ser uma ferramenta para ajudar o sistema imunológico contra a CoViD-19. Enquanto, Zadow et al. contribuíram com a análise a respeito das interações de coagulação com o efeito protetor do exercício físico para a CoViD-19. Nunn et al.(11) trouxeram profunda e abrangente análise a respeito da relação do estilo de vida - que trata do nível de atividade física e outros hábitos em saúde e do processo de envelhecimento com a saúde mitocondrial e as implicações para CoViD-19. Que foram citados nos itens prévios no presente estudo.

Resumidamente, a literatura indica que:

$>\mathrm{O}$ estilo de vida que promove a saúde mitocondrial, contribuindo para a manutenção do sistema imunológico, inclui a prática de exercício físico, dieta que inclui vegetais e redução calórica(11).

A intensidade do exercício físico é importante para melhorar a função endotelial, a imunidade e a saúde mental, e deve ser moderada, além de ser mantida a regularidade da prática $(39,49)$.

Hamer et al.(50), conduziram estudo longitudinal de coorte prospectivo em amostra populacional $(\mathrm{n}=387.109)$, no Reino Unido, no qual examinaram variáveis de estilo de vida em relação à internação hospitalar por CoViD-19. Os resultados foram que o risco relativo de ser hospitalizado pela doença foi maior entre os fisicamente inativos $(32 \%)$ e os fumantes 
$(42 \%)$. Entre os obesos o risco relativo para hospitalização por CoViD-19 foi maior do que o dobro $(2,05)$ em comparação com os não obesos(50).

No Brasil, o estudo de Vancini et al.(51) citado recentemente(52) o principal achado foi que houve associação de nível de atividade física com prevalência de sintomas de CoViD-19 com $(p=0,0012)$, conforme se observa na Tabela 1.

Tabela 1 - Prevalência de sintomas de CoViD-19 segundo nível de atividade física $(\mathrm{n}=1.726)$, estudo de Vancini et al. (51), conduzido no Brasil

\begin{tabular}{rrrc}
\hline $\begin{array}{l}\text { Nível de } \\
\text { atividade } \\
\text { física }\end{array}$ & $\boldsymbol{n}$ & $\begin{array}{c}\text { Freq. } \\
\text { Relativa } \\
\text { (\%) }\end{array}$ & $\begin{array}{c}\text { Prevalência } \\
\text { de } \\
\text { sintomas } \\
\text { de } \\
\text { CoViD-19 } \\
\text { (\%) }\end{array}$ \\
\hline \hline 1 & 620 & 35,9 & 30,6 \\
2 & 391 & 22,7 & 36,1 \\
3 & 425 & 24,6 & 19,4 \\
4 & 187 & 10,8 & 13,9 \\
5 & 103 & 6,0 & - \\
Total & 1.276 & 100 & - \\
\hline
\end{tabular}

Prevalência de sintomas de CoViD-19 segundo nível de atividade física. Teste de razão de verossimilhança $p$-valor $=\mathbf{0 , 0 0 1 2}$.

Considerando os níveis de atividade física, como categorias, tem-se: nível $1=$ insuficiente; nível 2 = muito baixo; nível 3 = baixo; nível $4=$ intermediário e nível $5=$ alto. $\mathrm{Na}$ amostra estudada $(\mathrm{n}=1.726)$, a maior parte apresentava nível insuficiente de atividade $(35,9 \%)$. E os níveis mais baixos de atividade física foram os que apresentaram as maiores prevalências de sintomas de CoViD-19. Conforme o nível de atividade física se elevou a prevalência diminuiu, sendo que o grupo com alto nível de atividade física $(n=103)$ não apresentou nenhum caso.

Yanuck et al.(53) apresentaram um estudo delineando uma estratégia baseada em evidências para melhorar os resultados clínicos em CoViD-19 utilizando uma abordagem imunofisiológica em fases para a doença desde a prevenção até a recuperação.
As quatro fases da CoViD-19(53):

1. Prevenção - o foco reside na eficiência da vigilância imunológica e na redução dos níveis basais de inflamação, com a finalidade melhorar o prognóstico, caso o indivíduo seja infectado;

2. Infecção - o foco é a atividade imunológica contra a infecção;

3. Inflamação crescente - o foco é em medidas anti-inflamatórias; e

4. Recuperação - o foco concentra-se na resolução da inflamação, inibindo a fibrose e outras formas de danos aos tecidos, reduzindo as perdas de função imune restaurando-a e reotimizando-a. Como foi observado que os pacientes recaíram na fase de inflamação crescente, é essencial que a vigilância clínica continue no que pode parecer ser a fase de recuperação.

O exercício físico deve estar presente atuando na fase de prevenção mantendo o sistema imunológico alerta, aprimorando a função endotelial, reduzindo o estresse oxidativo e diminuindo a inflamação. Nesse contexto, os autores propuseram que o exercício deve seguir as recomendações quanto à intensidade moderada para favorecer e aprimorar o sistema imune $(39,49)$.

\section{Conclusão}

O presente estudo apresentou uma breve revisão da literatura acerca da relação de exercício físico com aspectos de saúde, prevenção e recuperação da CoViD-19.

Os principais achados foram que $\mathrm{o}$ exercício promove a saúde mitocondrial, contribuindo para a manutenção do sistema imunológico. E a intensidade do exercício físico é importante para melhorar a função endotelial, a imunidade e a saúde mental, e deve ser realizada em intensidade moderada, além de ser mantida a regularidade da prática para que os benefícios orgânicos sejam alcançados. 
Nesse sentido, a literatura apresenta, até o momento, dois estudos mostrando associação inversa com a CoViD-19:

1. Quanto maior o nível de atividade física houve menos sintomas da doença, sendo que no nível mais alto não houve nenhum caso de sintomas de CoViD-19; e

2. Pessoas fisicamente inativas têm o risco $32 \%$ maior de ser hospitalizado por COViD-19.

Considerando que a obesidade aumenta o risco de agravamento da doença e que maiores níveis de atividade física têm mostrado efeito protetor para sintomas e internação por CoViD-19, recomenda-se a prática de exercícios regular tanto para a promoção da saúde, quanto para a prevenção da CoViD-19.

\section{Pontos fortes e limitações do estudo}

O ponto forte foi reunir o conhecimento quanto à relação entre exercício físico e nova doença que afeta o mundo em uma pandemia: a CoViD-19.

Uma limitação do estudo é que não se trata de uma revisão sistemática com a abrangência e delimitações amplas e bem definidas. Entretanto, a maioria dos estudos que integraram o presente estudo foi composta de estudos de revisão, além de ensaios clínicos e estudos epidemiológicos com amostra populacional, o que confere alta confiabilidade ao relato.

\section{Conclusão}

\section{Declaração de conflito de interesses}

Não há conflito de interesses em relação ao presente estudo.

\section{Declaração de financiamento}

Estudo conduzido sem financiamento.

\section{Referências}

1. Shibabaw T. Inflammatory Cytokine: IL17A Signaling Pathway in Patients Present with COVID-19 and Current Treatment Strategy. Journal of Inflammation Research. [Online] 2020;13: 673-680. Available from: doi:10.2147/JIR.S278335
2. Pamukçu B. Inflammation and thrombosis in patients with COVID-19: A prothrombotic and inflammatory disease caused by SARS coronavirus-2. Anatolian Journal of Cardiology. [Online] 2020;24(4): 224-234. Available from:

doi:10.14744/AnatolJCardiol.2020.56727

3. Ellul M, Varatharaj A, Nicholson TR, Pollak TA, Thomas N, Easton A, et al. Defining causality in COVID-19 and neurological disorders. Journal of Neurology, Neurosurgery, and Psychiatry. [Online] 2020;91(8): 811812. Available from: doi:10.1136/jnnp2020-323667

4. Carod-Artal FJ. Neurological complications of coronavirus and COVID-19. Revista De Neurologia. [Online] 2020;70(9): 311-322. Available from: doi:10.33588/rn.7009.2020179

5. Achar A, Ghosh C. COVID-19Associated Neurological Disorders: The Potential Route of CNS Invasion and Blood-Brain Relevance. Cells. [Online] 2020;9(11). Available from: doi: $10.3390 /$ cells9112360

6. Ng Kee Kwong KC, Mehta PR, Shukla G, Mehta AR. COVID-19, SARS and MERS: A neurological perspective. Journal of Clinical Neuroscience: Official Journal of the Neurosurgical Society of Australasia. [Online] 2020;77: 13-16. Available from: doi:10.1016/j.jocn.2020.04.124

7. Leonardi M, Padovani A, McArthur JC. Neurological manifestations associated with COVID-19: a review and a call for action. Journal of Neurology. [Online] 2020;267(6): 1573-1576. Available from: doi:10.1007/s00415-020-09896-z

8. Mehta P, McAuley DF, Brown M, Sanchez E, Tattersall RS, Manson JJ, et al. COVID-19: consider cytokine storm syndromes and immunosuppression. Lancet (London, England). [Online] 2020;395(10229): 1033-1034. Available from: doi:10.1016/S01406736(20)30628-0

9. Flogístico. Michaelis On-Line. [Online]. Available from: https://michaelis.uol.com.br/modernoportugues/busca/portugues- 
brasileiro/flog\%C3\%ADstico/ [Accessed: 28th January 2021]

10. Libby P, Lüscher T. COVID-19 is, in the end, an endothelial disease. European Heart Journal. [Online] 2020;41(32): 3038-3044. Available from: doi:10.1093/eurheartj/ehaa623

11. Nunn AVW, Guy GW, Brysch W, Botchway SW, Frasch W, Calabrese EJ, et al. SARS-CoV-2 and mitochondrial health: implications of lifestyle and ageing. Immunity \& Ageing: $I \& A$. [Online] 2020;17(1): 33. Available from: doi:10.1186/s12979-020-00204-x

12. Longo M, Bellastella G, Maiorino MI, Meier JJ, Esposito K, Giugliano D. Diabetes and Aging: From Treatment Goals to Pharmacologic Therapy. Frontiers in Endocrinology. [Online] Frontiers; 2019;10. Available from: doi:10.3389/fendo.2019.00045

[Accessed: 21st December 2020]

13. Cuschieri S, Grech S. COVID-19 and diabetes: The why, the what and the how. Journal of Diabetes and Its Complications. [Online] 2020;34(9): 107637. Available from: doi:10.1016/j.jdiacomp.2020.107637

14. Chen J, Kelley WJ, Goldstein DR. Role of Aging and the Immune Response to Respiratory Viral Infections: Potential Implications for COVID-19. Journal of Immunology (Baltimore, Md.: 1950). [Online] 2020;205(2): 313-320. Available from: doi:10.4049/jimmunol.2000380

15. Roman MA, Rossiter HB, Casaburi R. Exercise, ageing and the lung. European Respiratory Journal. [Online] European Respiratory Society; 2016;48(5): 14711486. Available from: doi:10.1183/13993003.00347-2016

16. Nystoriak MA, Bhatnagar A. Cardiovascular Effects and Benefits of Exercise. Frontiers in Cardiovascular Medicine. [Online] 2018;5. Available from: doi:10.3389/fcvm.2018.00135 [Accessed: 21st December 2020]

17. Niebauer J, Cooke JP. Cardiovascular effects of exercise: role of endothelial shear stress. Journal of the American College of Cardiology. [Online]
1996;28(7): 1652-1660. Available from: doi:10.1016/S0735-1097(96)00393-2

18. Stepp DW, Merkus D, Nishikawa Y, Chilian WM. Nitric oxide limits coronary vasoconstriction by a shear stressdependent mechanism. American Journal of Physiology. Heart and Circulatory Physiology. [Online] 2001;281(2): H796803. Available from: doi:10.1152/ajpheart.2001.281.2.H796

19. Swift DL, Johannsen NM, Lavie CJ, Earnest CP, Church TS. The Role of Exercise and Physical Activity in Weight Loss and Maintenance. Progress in cardiovascular diseases. [Online] 2014;56(4): 441-447. Available from: doi:10.1016/j.pcad.2013.09.012

20. Burtscher J, Burtscher M, Millet GP. (Indoor) isolation, stress, and physical inactivity: Vicious circles accelerated by COVID-19? Scandinavian Journal of Medicine \& Science in Sports. [Online] 2020;30(8): 1544-1545. Available from: doi: $10.1111 /$ sms. 13706

21. Jiménez-Pavón D, Carbonell-Baeza A, Lavie CJ. Physical exercise as therapy to fight against the mental and physical consequences of COVID-19 quarantine: Special focus in older people. Progress in Cardiovascular Diseases. [Online] 2020;63(3): 386-388. Available from: doi:10.1016/j.pcad.2020.03.009

22. Pedersen BK, Hoffman-Goetz L. Exercise and the immune system: regulation, integration, and adaptation. Physiological Reviews. [Online] 2000;80(3): 10551081. Available from: doi:10.1152/physrev.2000.80.3.1055

23. Mohamed AA, Alawna M. Role of increasing the aerobic capacity on improving the function of immune and respiratory systems in patients with coronavirus (COVID-19): A review. Diabetes \& Metabolic Syndrome. [Online] 2020;14(4): 489-496. Available from: doi:10.1016/j.dsx.2020.04.038

24. Warburton DER, Nicol CW, Bredin SSD. Health benefits of physical activity: the evidence. CMAJ: Canadian Medical Association journal = journal de l'Association medicale canadienne. [Online] 2006;174(6): 801-809. Available from: doi:10.1503/cmaj.051351 
25. Maiorana A, O'Driscoll G, Taylor R, Green D. Exercise and the nitric oxide vasodilator system. Sports Medicine (Auckland, N.Z.). [Online] 2003;33(14): 1013-1035. Available from: doi:10.2165/00007256-20033314000001

26. Green WD, Beck MA. Obesity Impairs the Adaptive Immune Response to Influenza Virus. Annals of the American Thoracic Society. [Online] American Thoracic Society - AJRCCM; 2017;14(Supplement 5): S406-S409. Available from: doi:10.1513/AnnalsATS.201706-447AW

27. Ghisi GL de M, Durieux A, Pinho R, Benetti M. Physical exercise and endothelial dysfunction. Arquivos Brasileiros de Cardiologia. [Online] Arquivos Brasileiros de Cardiologia; 2010;95(5): e130-e137. Available from: doi:10.1590/S0066-782X2010001500025

28. Paszkowiak JJ, Dardik A. Arterial wall shear stress: observations from the bench to the bedside. Vascular and Endovascular Surgery. [Online] 2003;37(1): 47-57. Available from: doi: $10.1177 / 153857440303700107$

29. Pries Axel R., Reglin Bettina, Secomb Timothy W. Remodeling of Blood Vessels. Hypertension. [Online] American Heart Association; 2005;46(4): 725-731. Available from: doi:10.1161/01.HYP.0000184428.16429. be

30. Souilhol C, Serbanovic-Canic J, Fragiadaki M, Chico TJ, Ridger V, Roddie H, et al. Endothelial responses to shear stress in atherosclerosis: a novel role for developmental genes. Nature Reviews. Cardiology. [Online] 2020;17(1): 52-63. Available from: doi:10.1038/s41569-0190239-5

31. Green DJ, Hopman MTE, Padilla J, Laughlin $\mathrm{MH}$, Thijssen DHJ. Vascular Adaptation to Exercise in Humans: Role of Hemodynamic Stimuli. Physiological Reviews. [Online] 2017;97(2): 495-528. Available doi:10.1152/physrev.00014.2016

32. Green DJ, Smith KJ. Effects of Exercise on Vascular Function, Structure, and Health in Humans. Cold Spring Harbor Perspectives in Medicine. [Online]
2018;8(4). Available from: doi:10.1101/cshperspect.a029819

33. Xie J-H, Li Y-Y, Jin J. The essential functions of mitochondrial dynamics in immune cells. Cellular \& Molecular Immunology. [Online] 2020;17(7): 712721. Available from: doi:10.1038/s41423-020-0480-1

34. Fission. Cambridge Dictionary. [Online]. Available from: https://dictionary.cambridge.org/dictiona ry/english-portuguese/fission [Accessed: 29th January 2021]

35. Mattson MP. Hormesis defined. Ageing Research Reviews. [Online] 2008;7(1): 1$7 . \quad$ Available from: doi:10.1016/j.arr.2007.08.007

36. Tapia PC. Sublethal mitochondrial stress with an attendant stoichiometric augmentation of reactive oxygen species may precipitate many of the beneficial alterations in cellular physiology produced by caloric restriction, intermittent fasting, exercise and dietary phytonutrients: 'Mitohormesis' for health and vitality. Medical Hypotheses. [Online] 2006;66(4): 832-843. Available from: doi:10.1016/j.mehy.2005.09.009

37. Ristow M, Zarse K. How increased oxidative stress promotes longevity and metabolic health: The concept of mitochondrial hormesis (mitohormesis). Experimental Gerontology. [Online] 2010;45(6): 410-418. Available from: doi:10.1016/j.exger.2010.03.014

38. Wu J, Weisshaar N, Hotz-Wagenblatt A, Madi A, Ma S, Mieg A, et al. Skeletal muscle antagonizes antiviral CD8+ T cell exhaustion. Science Advances. [Online] American Association for the Advancement of Science; 2020;6(24): eaba3458. Available from: doi:10.1126/sciadv.aba3458

39. da Silveira MP, da Silva Fagundes KK, Bizuti MR, Starck É, Rossi RC, de Resende e Silva DT. Physical exercise as a tool to help the immune system against COVID-19: an integrative review of the current literature. Clinical and Experimental Medicine. [Online] 2020; Available from: doi:10.1007/s10238-02000650-3 [Accessed: 10th December 2020] 
40. Danesh J, Wheeler JG, Hirschfield GM, Eda S, Eiriksdottir G, Rumley A, et al. Creactive protein and other circulating markers of inflammation in the prediction of coronary heart disease. The New England Journal of Medicine. [Online] 2004;350(14): 1387-1397. Available from: doi:10.1056/NEJMoa032804

41. Pradhan AD, Manson JE, Rifai N, Buring JE, Ridker PM. C-reactive protein, interleukin 6 , and risk of developing type 2 diabetes mellitus. JAMA. [Online] 2001;286(3): 327-334. Available from: doi:10.1001/jama.286.3.327

42. Khandaker GM, Dantzer R, Jones PB. Immunopsychiatry: important facts. Psychological Medicine. [Online] 2017;47(13): 2229-2237. Available from: doi:10.1017/S0033291717000745

43. Hamer M, Endrighi R, Poole L. Physical activity, stress reduction, and mood: insight into immunological mechanisms. Methods in Molecular Biology (Clifton, N.J.). [Online] 2012;934: 89-102. Available from: doi:10.1007/978-162703-071-7_5

44. Duric V, Clayton S, Leong ML, Yuan LL. Comorbidity Factors and Brain Mechanisms Linking Chronic Stress and Systemic Illness. Neural Plasticity. [Online] 2016;2016: 5460732. Available from: doi:10.1155/2016/5460732

45. Carek PJ, Laibstain SE, Carek SM. Exercise for the treatment of depression and anxiety. International Journal of Psychiatry in Medicine. [Online] 2011;41(1): 15-28. Available from: doi:10.2190/PM.41.1.c

46. Paluska SA, Schwenk TL. Physical activity and mental health: current concepts. Sports Medicine (Auckland, N.Z.). [Online] 2000;29(3): 167-180. Available from: doi:10.2165/00007256200029030-00003

47. Hosker DK, Elkins RM, Potter MP. Promoting Mental Health and Wellness in Youth Through Physical Activity, Nutrition, and Sleep. Child and Adolescent Psychiatric Clinics of North America. [Online] 2019;28(2): 171-193. Available from: doi:10.1016/j.chc.2018.11.010
48. Paolucci EM, Loukov D, Bowdish DME, Heisz JJ. Exercise reduces depression and inflammation but intensity matters. Biological Psychology. [Online] 2018;133: 79-84. Available from: doi:10.1016/j.biopsycho.2018.01.015

49. Zadow EK, Wundersitz DWT, Hughes DL, Adams MJ, Kingsley MIC, Blacklock HA, et al. Coronavirus (COVID-19), Coagulation, and Exercise: Interactions That May Influence Health Outcomes. Seminars in Thrombosis and Hemostasis. [Online] 2020;46(7): 807-814. Available from: doi:10.1055/s-0040-1715094

50. Hamer M, Kivimäki M, Gale CR, Batty GD. Lifestyle Risk Factors for Cardiovascular Disease in Relation to COVID-19 Hospitalization: A Community-Based Cohort Study of 387,109 Adults in UK. medRxiv. [Online] 2020; Available from: doi: $10.1101 / 2020.05 .09 .20096438$

[Accessed: 21st December 2020]

51. Vancini RL, Camargo-Neto L, de Lira CAB, Andrade MS, Viana RB, Nikolaidis PT, et al. Physical Activity and Sociodemographic Profile of Brazilian People during COVID-19 Outbreak: An Online and Cross-Sectional Survey. International Journal of Environmental Research and Public Health. [Online] 2020;17(21). Available from: doi:10.3390/ijerph17217964

52. Corpo Editorial. Atividade física $\mathrm{e}$ sociodemografia em 1.726 brasileiros durante a pandemia de CoViD-19: resenha apresentando o artigo de Vancini et al. Revista de Educação Física / Journal of Physical Education. [Online] 2020;89(3): 176-179. Available from: doi:10.37310/ref.v89i3.2709

53. Yanuck SF, Pizzorno J, Messier H, Fitzgerald KN. Evidence Supporting a Phased Immuno-physiological Approach to COVID-19 From Prevention Through Recovery. Integrative Medicine (Encinitas, Calif.). 2020;19(Suppl 1): 835.

54. Ashor AW, Lara J, Siervo M, CelisMorales C, Oggioni C, Jakovljevic DG, et al. Exercise modalities and endothelial function: a systematic review and doseresponse meta-analysis of randomized controlled trials. Sports Medicine 
(Auckland, N.Z.). [Online] 2015;45(2):

279-296. Available from:

doi:10.1007/s40279-014-0272-9 